\title{
Sostenibilidad y proyectos sostenibles: Estudio bibliométrico
}

\section{Sustainability and sustainable projects: Bibliometric study}

\author{
Omaira Manzano - Durán ${ }^{1}$, Marta Milena Peñaranda - Peñaranda², \\ Juan Carlos Luna - Quintero ${ }^{3}$ \\ ${ }^{1}$ Grupo de investigación GRINDES, Universidad Nacional Abierta y a Distancia, Colombia \\ ORCID: http://orcid.org/0000-0002-2715-8903,Email: omaira.manzano@unad.edu.co \\ ${ }^{2}$ Grupo de investigación GIDSE, Universidad Francisco de Paula Santander Ocaña, Colombia \\ ORCID: https://orcid.org/0000-0002-9257-9245, Email: mmpenarandap@ufpso.edu.co \\ ${ }^{3}$ Grupo de investigación GRINDES, Universidad Nacional Abierta y a Distancia, Colombia \\ ORCID: https://orcid.org/0000-0002-4076-4596, Email: juan.luna@unad.edu.co
}

Cómo citar: Manzano - Durán, O., Peñaranda - Peñaranda, M. M., \& Luna - Quintero, J. C. (2021). Sostenibilidad y proyectos sostenibles: Estudio bibliométrico. Revista Científica Profundidad Construyendo Futuro, 14(14), 15-24. https://doi.org/10.22463/24221783.3160

Recibido: 16 de Julio de 2020 / Aprobado: 18 de Octubre de 2020

\begin{abstract}
Resumen
La presente investigación responde a un enfoque cuantitativo de tipo longitudinal descriptivo con el fin de construir un análisis bibliométrico sobre la sostenibilidad en los proyectos, evidenciando el estado de la ciencia en esta importante área. Mediante el empleo de la base de datos Scopus, Excel y el software VOSviewer se analizaron indicadores de los 550 documentos encontrados desde el año 1990 hasta el 2020. Como resultado se encontró que la calidad y cantidad de los documentos al igual que su estructura es alta, sin embargo, se requieren mejorar las publicaciones de libros en esta área. A partir del mapa de co-ocurrencia realizado se logra identificar 3 clúster o categorías analizadas: Sostenibilidad, desarrollo sostenible y proyectos sostenibles.
\end{abstract}

Palabras claves: Bibliometría, Desarrollo sostenible, Proyectos sostenibles, Sostenibilidad.

\begin{abstract}
The present research responds to a descriptive longitudinal quantitative approach in order to construct a bibliometric analysis on the sustainability of the projects, evidencing the state of science in this important area. Through the use of the Scopus database, Excel and the VOSviewer software, indicators of the 550 documents found from 1990 to 2020 were analyzed. As a result, it was found that the quality and quantity of the documents as well as their structure is high. However, there is a need to improve book publications in this area. From the map of co-occurrence carried out, it is possible to identify 3 clusters or categories analyzed: Sustainability, sustainable development and sustainable projects.
\end{abstract}

Key words: Bibliometrics, Sustainable development, Sustainable projects, Sustainability. 


\section{Introducción}

El desarrollo económico a nivel mundial ha llevado a que la humanidad haga un uso indiscriminado de los recursos. Sin embargo, la sociedad ha tomado conciencia de la necesidad del desarrollo sostenible en todos los proyectos, iniciativas empresariales, pequeñas y grandes empresas que respondan a la triple cuenta de resultados, donde no solo prima lo económico sino el impacto social y ambiental.

Durante los últimos años el término desarrollo sostenible ha sido un área que ha sido estudiado no solo por académicos o la comunidad científica, sino que también es preocupación creciente de organismos gubernamentales. Se hace necesario ante las crisis manifiestas lograr un desarrollo que supla las necesidades del presente sin comprometer la capacidad de las generaciones futuras (Porras Barajas, 2017).

La sostenibilidad debe ser una responsabilidad mundial de todos los individuos y no solo de las grandes empresas. Por lo tanto, una adecuada gestión de proyectos debe estar direccionada a mejorar la calidad de vida no solo de las generaciones presentes sino de las futuras (Folgado Galache, 2016).

El presente estudio tiene como fin presentar los resultados de un análisis bibliométrico en el área de los proyectos sostenibles que sirva como antecedente para futuras investigaciones que deseen conocer el estado de la ciencia y los logros alcanzados por los académicos centrados en esta importante temática. Las herramientas empleadas fue la base de datos Scopus, Excel junto con el software VOSviewer que permitió identificar número de publicaciones, área de publicación, tipos de documento publicado, país de origen de las publicaciones, autores y su filiación, palabras claves entre otros. A partir del análisis de coocurrencia se encuentran tres clúster $o$ categorías de análisis: Sostenibilidad, desarrollo sostenible y proyectos sostenibles.

\section{Metodología}

El paradigma seleccionado para la presente investigación fue Cuantitativo no experimental de corte Longitudinal de los años 1990-2020. La base de datos seleccionada fue Scopus por su relevancia en el mundo académico y sus capacidades para el análisis de grandes cantidades de información. Las fases de la investigación se presentan a continuación:

Fase 1. Ingreso en la base de datos Scopus de la ecuación de búsqueda "Proyectos Sostenibles" OR "Sustaibable Project" durante los años 1990-2020

Fase 2. Se encuentran 550 documentos que se analizaron para identificar los indicadores bibliométricos

Fase 3. El conjunto de metadatos que se extrajeron de la base de datos de Scopus se procesan y analizan en el software VOSviewer. Los datos que se exportan bajo el formato CSV.

Fase 4. Se hace el análisis de la información y los clústeres acordes a los resultados y estadísticas encontradas.

\section{Resultados}

Al introducir la ecuación de búsqueda en la base de datos Scopus: Proyectos Sostenibles" OR "Sustaibable Project" se encuentran 550 documentos. Como se 
aprecia en la Figura 1. los primeros 2 documentos que se hacen referencia a los proyectos sostenibles se encuentran en el año 1990, evolucionando de una manera exponencial hasta el año 2020 con 79 documentos sobre esta importante temática. Se aprecia un pico en el año 2013, periodo que se encuentra en la señalada "década de la educación para un futuro sustentable 20052014"

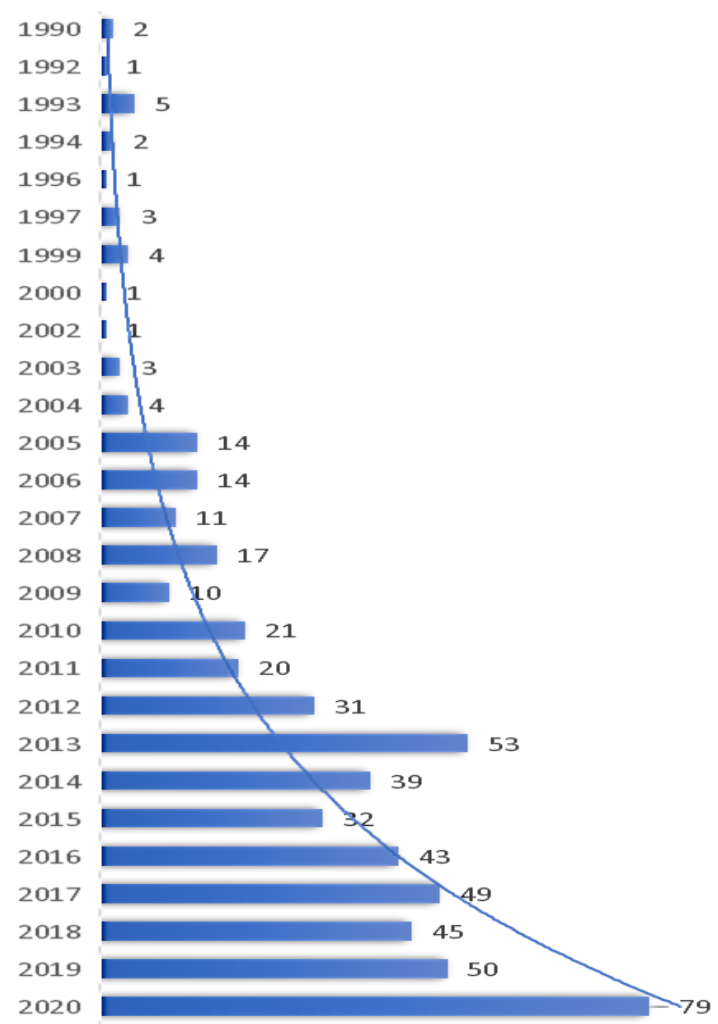

Figura 1. Documentos por año. Fuente: Los autores a partir de la base de datos Scopus.

En cuanto a la difusión del conocimiento, se puede apreciar que de los 555 documentos encontrados en la base de datos Scopus el $55 \%$ son artículos científicos seguido de un $31.5 \%$ de conferencias y un $6.7 \%$ capítulos de libro. Se aprecia poca productividad en libros sobre proyectos sostenibles con solo un $1.1 \%$ (Ver figura 2).

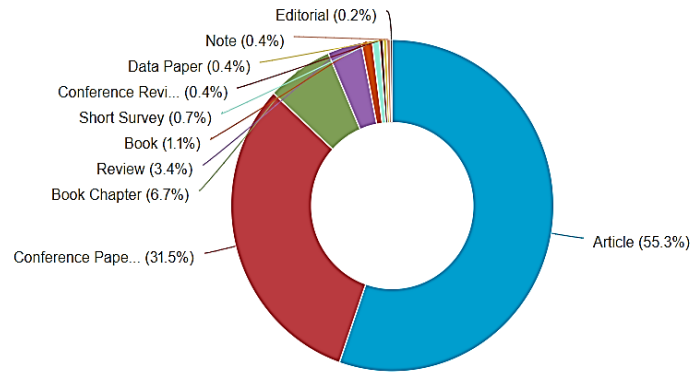

Figura 2. Difusión del conocimiento. Fuente: Los autores a partir de la base de datos Scopus.

La productividad anteriormente mencionada está centrada en un $23.6 \%$ en el área de ingeniería, seguida por un $16.1 \%$ en el área ambiental y un $14.3 \%$ en áreas sociales. Se aprecia una cifra representativa de publicaciones en proyectos sostenibles en el área de negocios y administración con un $11.7 \%$ (Ver figura 3).

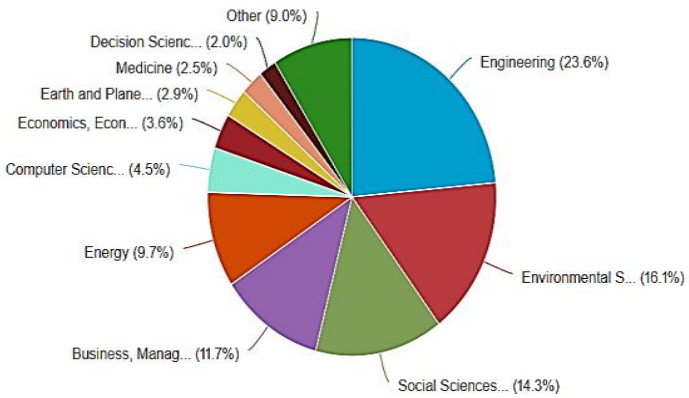

Figura 3. Documentos por áreas de conocimiento. Fuente: Los autores a partir de la base de datos Scopus.

Asimismo, se pueden observar en la figura 4. Los diez investigadores que más publicaciones tienen sobre el tema. Siendo los tres primeros Mohagheghi, Mousavi y Schipper con cinco publicaciones cada uno. En este sentido, estos diez investigadores solo publican el $7 \%$ de la producción científica en proyectos sostenibles. Mohagheghi, Vahid Mousavi, S. Meysam tienen la misma filiación institucional con la Shahed University, Tehran, Iran. 


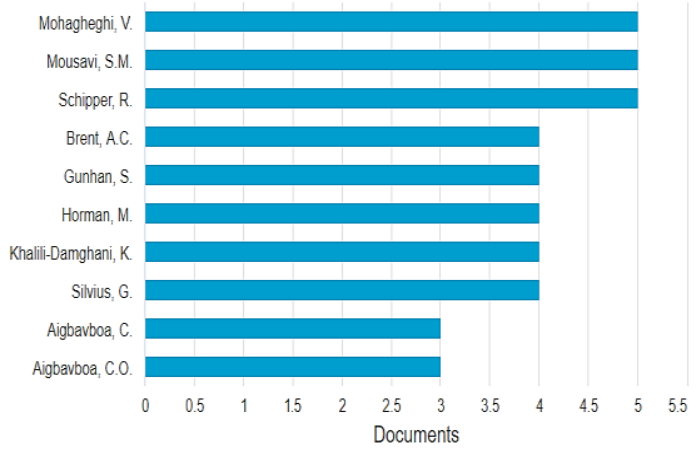

Figura 4. Documentos por autor. Fuente: Los autores a partir de la base de datos Scopus.

Por otro lado, haciendo referencia a la afiliación, se aprecia que las instituciones o universidades que más aportan en el área de proyectos sostenibles son: Universidad de Johannesburg, universidad de Pennsylvania State y LOI University of Applied Sciences (ver figura 5).

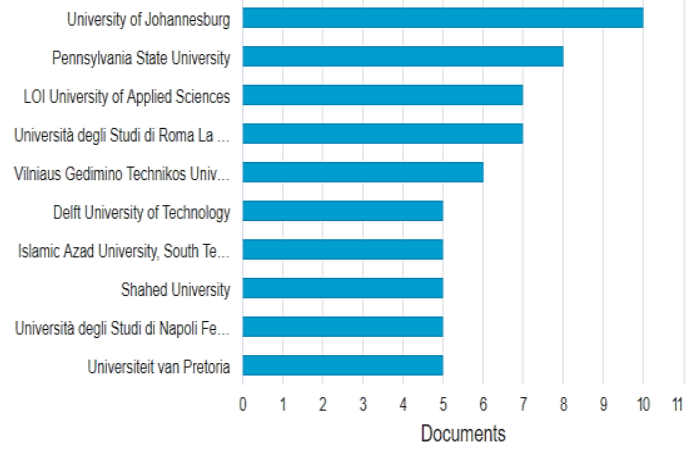

Figura 5. Documentos por afiliación. Fuente: Los autores a partir de la base de datos Scopus.

Además, en la figura 6. Se aprecian los países que dieron origen a los documentos publicados sobre proyectos sostenibles. Encontrándose en primer lugar Estados Unidos responsable del $24 \%$ de dichas publicaciones, seguido de United Kingdom y China cada uno con un 6\%. Es necesario resaltar que se encuentra Brazil como el único país suramericano en la lista.

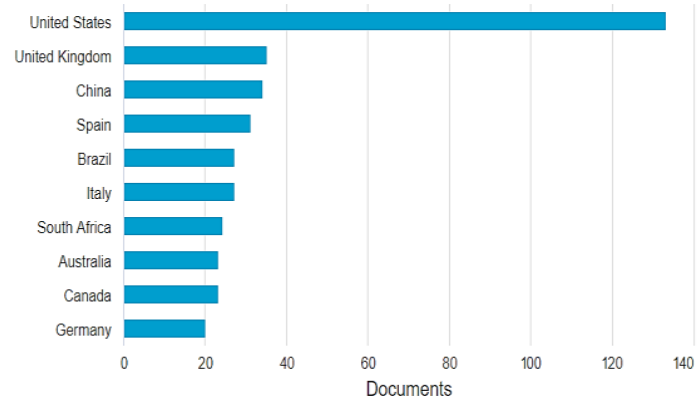

Figura 6. Países de origen de los autores. Fuente: Los autores a partir de la base de datos Scopus.

Indicador de Co-ocurrencia. El análisis de co-ocurrencia efectuado en el Software VOSViewer a partir de las 3955 palabras claves detectadas en la base de datos Socups, toma como unidad de análisis todas las palabras, seleccionando el umbral de coocurrencia mínima de 10 palabras. Es conveniente aclarar que el programa selecciona las palabras claves con la mayor fuerza de enlace total. Por lo tanto, en este mapa de coocurrencias sobre proyectos sostenibles se encuentran 3 clúster: Sostenibilidad, Desarrollo sostenible y Proyectos sostenibles que se explican a continuación.

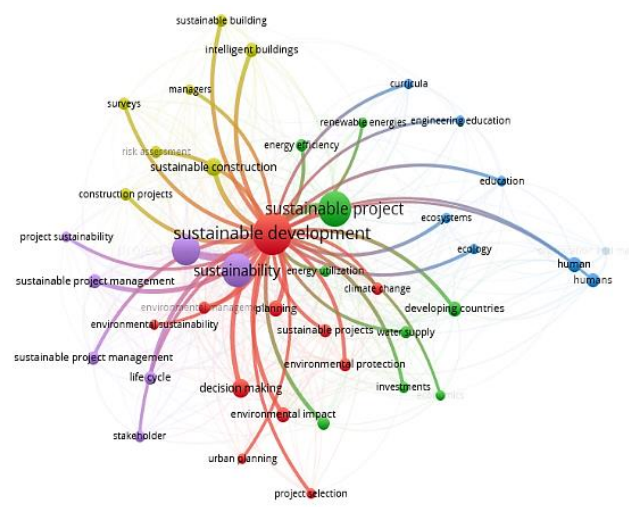

Figura 7. Análisis de Co-ocurrencia. Fuente: Los autores a partir de la base de datos Scopus.

Sostenibilidad y desarrollo sostenible. Los aspectos ambientales como el desarrollo sostenible, la responsabilidad social, la ética y la importancia de la cadena de suministros 
han tomado fuerza mundial debido a la preocupación de la humanidad por el cambio climático que se está viviendo. Es allí donde surge una postura renovada por la implementación de prácticas de negocios sostenibles, los cuales buscan garantizar procesos producción que minimicen el impacto ambiental, siendo receptivos y sensibles con las comunidades donde se desarrolla la actividad comercial de las empresas.

Teniendo en cuenta lo expuesto, el concepto de sostenibilidad toma un valor central dentro de la política de la Gerencia de Proyectos Verdes o GPM (por sus siglas en inglés, Green Project Management), al igual que la gestión sostenible. Por ende, "la sostenibilidad es la capacidad de un sistema o de un proceso de mantenerse a sí mismo a lo largo de un periodo amplio de tiempo" (Moldan y Dahl, 2007).

Para (Gareis, Huemann, Martinuzzi, Weninger, y Sedlacko, 2013), desde la perspectiva del Project Management Institute (PMI), desarrollo sostenible se concibe como una nueva postura paradigmática de la gestión de proyectos que encamina a las organizaciones en la compleja ruta $y$ dinámica de sus proyectos y programas. El PMI establece que los principios del desarrollo sostenible (económicos, ecológicos y sociales) son primordiales a ser tenidos en cuenta en la formulación y gestión de proyectos con el ánimo de lograr excelentes resultados y con esto direccionar la organización hacia la calidad.

La concepción de "desarrollo sostenible" es relativamente nueva, fue la Comisión Mundial de Ambiente y Desarrollo con el nombre "Nuestro Futuro Común" que en el año 1987 elabora la primera definición, en la cual se describe al desarrollo como la forma de enfrentar los requerimientos actuales sin comprometer "la habilidad de las futuras generaciones para afrontar sus propias necesidades" (World Commission on Environment and Development [WCED], 1987, p.43). Por lo tanto, para que exista desarrollo tal como se plantea es necesario la igualdad de derechos de las naciones y personas con el ánimo de poder crecer y florecer tanto en el presente como en el futuro de la sociedad (Robertson, 2017).

A pesar de entenderse como una definición sencilla, el Desarrollo Sostenible se posee especificaciones que la convierten en un proceso algo complejo, debido a que el concepto está orientado a la armonización y crecimiento tanto social como económico de las sociedades con su entorno ecológico y ambiental, con lo que se busca garantizar la existencia de la especie humana protegiendo el planeta de daños ambientales.

Esta filosofía que busca brindar la oportunidad de una vida digna para la humanidad está fundamentada en tres principios rectores, Ambiente, Economía y Equidad (conocidos en inglés como las tres E, environment, economy and equity), así como también las tres $\mathrm{P}$ (por sus iniciales en inglés): Personas, Planeta y Ganancias (People, Planet and Profit), las cuales identifican una "Línea Base según el término acuñado por John Elkington hacia finales del siglo pasado. De esta manera el desarrollo sostenible incluye tres dimensiones: la sostenibilidad ecológica, las oportunidades económicas y la inclusión social” (Robertson, 2017, p.3).

Es evidente como el problema de sostenibilidad requiere del compromiso de todos y es allí donde organismos multilaterales como la Organización de las Naciones Unidas - ONU ha venido promoviendo cumbres para tratar el tema y 
lograr concientizar a las organizaciones en la búsqueda de "estrategias corporativas: prácticas de producción, modelos de negocio, servicios, procesos, recursos, e inclusive, los indicadores de gestión deben incorporar el marco de la sostenibilidad" (Silvius y Tharp, 2013).

Por ende, la sociedad y sus organizaciones deben integrar dentro de sus prácticas productivas aspectos socio-económicos y medioambientales, direccionados hacia la sostenibilidad del entorno como aporte de valor al crecimiento social, con el ánimo de minimizar el impacto generado por las actividades realizadas en los sus grupos de interés.

A continuación, en la Figura 8 se aprecia el concepto de sostenibilidad desde la mirada de diversos autores.

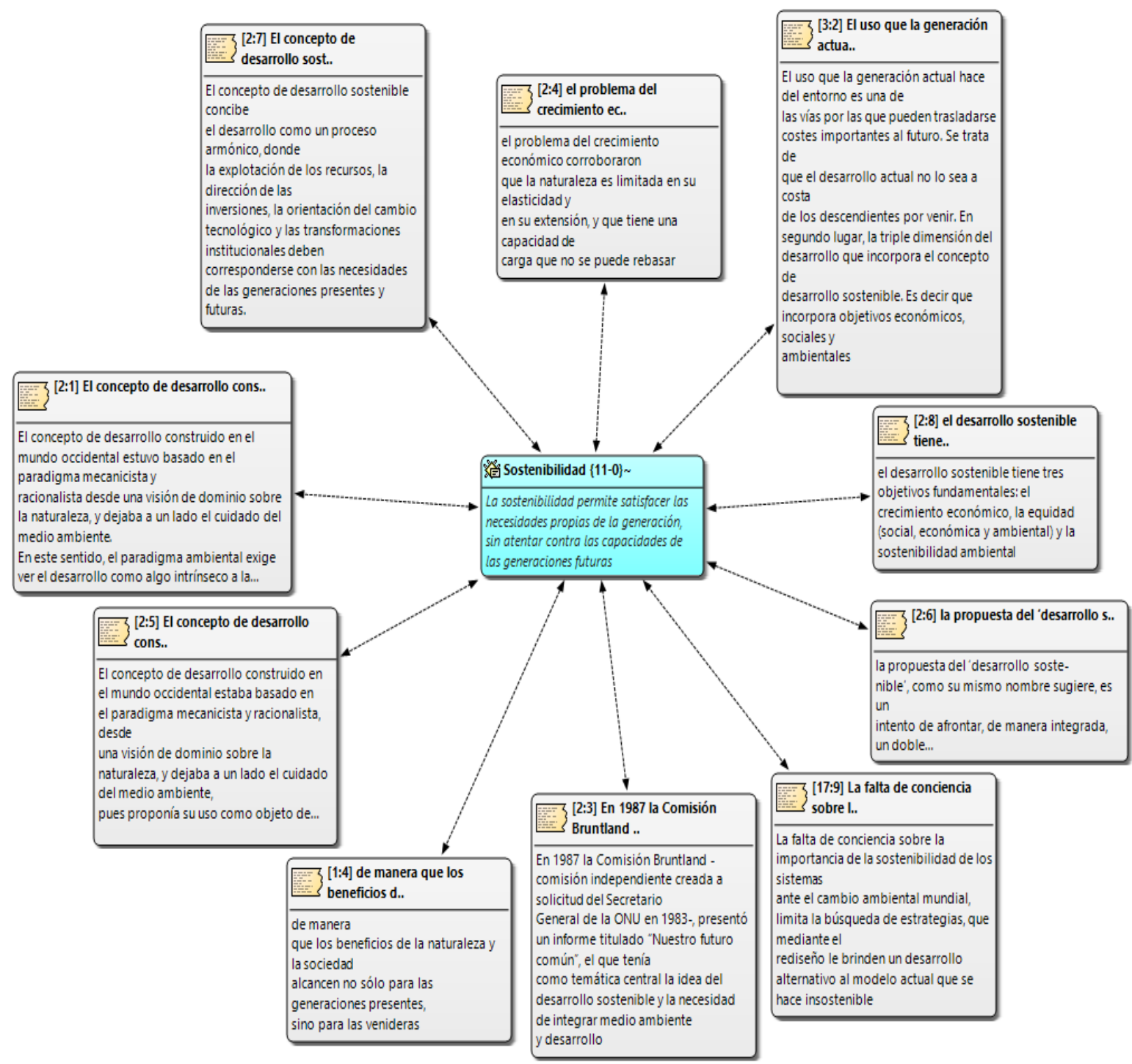

Figura 8. Concepto de Sostenibilidad. Fuente: (Luna Quintero, Ortega Marcón, y Manzano Duran, 2018). 
Proyectos sostenibles. El reto para las generaciones y organizaciones futuras deberá estar enfocado en proteger el desarrollo económico e industrial en las sociedades, afrontando los retos ambientales que se avecinan. Por lo tanto, para el siglo XXI las organizaciones deben enfocar la dirección de sus proyectos tanto empresariales como sociales en la adaptación de las mismas a las nuevas condiciones, y es allí donde los procesos y la evaluación de los proyectos se convierten en áreas fundamentales para el éxito de la organización, sin embrago, esta tarea no será nada fácil, debido a el enfoque de gestión sostenible es relativamente nuevo (Fomina y Apenko, 2019).

Sejenovich (2020) señala que la planificación de los proyectos no debe estar solamente enfocada hacia la rentabilidad económica, sino que la inclusión del cuidado del medio ambiente y el bienestar social son parte integral de los mismos, con lo que se tienen mejores posibilidades de obtener recursos para su financiación. En este sentido, Rodríguez Castilla, Peñaranda Peñaranda, y Manzano Duran, (2020) afirman que las organizaciones dentro de su compromiso social deben elaborar proyectos encaminados a la mitigación de los daños ambientales, con lo cual se busca promover proyectos productivos que favorezcan las condiciones óptimas para la existencia de futuras generaciones.

Por ende, la búsqueda del éxito de los proyectos debe ir más allá de la simple búsqueda de los beneficios económicos, aspectos prácticos como la cultura, donde se puede involucrar de forma práctica a los grupos de interés (stakeholders) cumplen un factor determinante y clave en el éxito de los proyectos, no solo en su desarrollo, sino también en la valoración de los resultados en el mediano y largo plazo (Motoa, 2015).
La preocupación por los efectos negativos que la sociedad y las organizaciones están ocasionado al planeta y su parte ambiental, está cambiando la forma de direccionar la formulación de los proyectos, generando estrategias con tendencias hacia lo ambiental que buscan una sostenibilidad como parte primordial de los proyectos. Dentro se estas tendencias se puede mencionar la metodología de gestión de proyectos Green Proyect Management, la cual está enfocada en la gestión de los proyectos de forma integral donde la economía sea rentable, el ambiente sea sostenible y la organización sea socialmente responsable.

Debido a que en épocas anteriores los proyectos solo veían su objetivo en el factor económico, con las consecuencias medioambientales generadas por la humanidad al planeta, la forma de ver los proyectos de manera sostenible ha venido mejorando, es así como en la actualidad desde la ideación del proyecto ya se piensa en estrategias de viabilidad que involucra los componentes económicos, sociales y ambientales, los cuales generan un equilibrio constante para el éxito final (Plasencia Soler, Marrero Delgado, Bajo Sanjuán, y Nicado García, 2018). Desde el punto de vista de la sostenibilidad los proyectos deben cumplir con los procesos planeados de factores económicos, sociales y ambientales, los cueles son relevantes para lograr el bienestar de los grupos de interés y el mínimo impacto negativo al medio ambiente (Porras Barajas, 2017).

Es así como, la gestión de proyectos sostenibles debe considerar ciertos aspectos que son claves para el desarrollo del mismo, los cuales son mencionados en la Tabla 1. 
Tabla 1. Aspectos claves en la gestión de proyectos

Aspectos Descripción

Materiales

Son necesarios para la ejecución del proyecto, pero estos deben afectar lo menos posible al ambiente, lo que los convierte en amigables con el planeta, de igual forma se debe lograr que estos puedan estar dentro del ámbito de la economía circular.

Productos químicos

La mínima utilización de estos en el desarrollo del proyecto es un factor determinante dentro de la sustentabilidad, pero de igual forma su uso debe estar dentro de los estándares para no afectar la salud humana, al igual que posean un manejo ambiental sostenible.

\section{Recursos naturales}

Economía circular

Stakeholders internos
El grado de destrucción actual de los recursos naturales es tan alto, que los proyectos deben enfocarse en lo posible en la utilización de energías limpias o alternativas, buscando contribuir a la mejora del cambio climático y esto se logra con la optimización de los procesos productivos siendo eficientes en el uso de los recursos.
Sociedad y partes interesadas
Se define como sistema de aprovechamiento donde los recursos son reducidos, aprovechados y reutilizados. Esta filosofía aboga por la utilización de materiales biodegradables que puedan volver al ciclo natural del ambiente sin causar ningún impacto negativo a los ecosistemas.

Los clientes internos son parte fundamental de las organizaciones, por lo tanto, estas deben proveer puestos de trabajo dignos, con los cuales se logran mejores relaciones laborales. Además, logra bienestar, formación y comunicación todo esto bajo un esquema de entrenamiento y divagación constate.

Al igual que los clientes internos, la sociedad como parte de los grupos de interés debe estar al tanto de lo que la organización está planteando, por ello la comunicación y apoyo constante con el entorne juega un papel fundamental dentro de la responsabilidad social de los proyectos. Esto contribuye a conocer las necesidades del entorno y su posición frente a la ejecución del proyecto.

Ética

La trasparencia dentro del desarrollo de los proyectos se convierte en eje central de la credibilidad ante las partes interesadas, por ello se requiere la no utilización de prácticas deshonestas como, corrupción, competencias desleales, soborno entre otros.

Fuente: Los autores a partir de la bibliografía citada. 


\section{Conclusiones}

Se concluye que los documentos publicados sobre proyectos sostenibles tienen un crecimiento exponencial desde el año 1990 al 2020. Los estudios se han centrado en su mayoría en el área de ingeniería con un $23.6 \%$, seguido por el área ambiental en un 23.6\%. Sin embargo, empiezan a emerger estudios en el área de negocios y administración con un $11.7 \%$. El país donde más publicaciones se han realizado sobre la temática es Estados Unidos responsable del $24 \%$ de dichos documentos.

Los proyectos sostenibles son un tema que ha copado la agenda de diversos países por su relevancia en la preservación del ambiente. Se hace necesario que los gestores de proyectos asuman este reto y minimicen el impacto de sus operaciones mediante el empleo de energías limpias, materiales biodegradables y la economía circular para un adecuado aprovechamiento y reutilización de los recursos.

\section{Referencias}

Folgado Galache, L. (2016). Plan de Sostenibilidad. Obtenido de http://iesataulfoargenta.es/ataulfosost enible/wpcontent/uploads/2016/11/Plan-deSostenibilidad-IES-Ataulfo-Argenta2016-2017.pdf

Fomina, Y., y Apenko, S. (2019). Green project management: Balancing of sustainable development principles. En M. Ibrahimov, A. Aleksic, \& D. Dukic (Ed.), 37th International Scientific Conference on Economic and Social Development - "Socio Economic Problems of Sustainable
Development", (págs. 1496-1501). Bakú. Obtenido de https://www.researchgate.net/profile/ AvazAlakbarov/publication/341741565_F INANCIAL_STABILITY_ASSESS MENT_OF_THE_BANKING_SECT OR_ON_THE_BASIS_OF_COMPO SITE_INDEX/links/5ed1676d45851 529451bbe81/FINANCIALSTABILITY-ASSESSMENT-OFTHE-BANKING-SECTOR-ONTHE-BASIS

Gareis, R., Huemann, M., Martinuzzi, A., Weninger, C., y Sedlacko, M. (2013). Project Management and Sustainable Development Principles. Newtown Square, Pennsylvania: Project Management Institute, Inc. Obtenido de https://www.pmi.org//media/pmi/documents/public/pdf/res earch/research-summaries/gareis_pm-and-sustainable-development.pdf

Luna Quintero, J. C., Ortega Marcón, Y. E., y Manzano Duran, O. (2018). Indicadores de sostenibilidad en la gestión de proyectos. Obtenido de https://repository.unad.edu.co/bitstrea $\mathrm{m} /$ handle/10596/25180/juan.luna.pdf ?sequence $=1$ \&isAllowed $=\mathrm{y}$

Moldan, B., y Dahl, A. L. (2007). SCOPE 67: Sustainability Indicators: A Scientific Assessment. Challenges to Sustainability Indicators, 1-24. (T. Hak, B. Moldan, \& A. L. Dahl, Edits.) Washington-Covelo-London: Island Press. Obtenido de https://www.researchgate.net/publicat ion/258261619_Sustainability_Indica tors_A_Scientific_Assessment_SCO PE_67 
Motoa, G. (2015). Medición del éxito en los proyectos, una revisión de la literatura. Revista Ingenium, 9(25), 11-25. doi:10.21774/ing.v9i25.584

Plasencia Soler, J. A., Marrero Delgado, F., Bajo Sanjuán, A. M., y Nicado García, M. (2018). Modelos para evaluar la sostenibilidad de las organizaciones. Revista Estudios Gerenciales, 34(146), 63-73. doi:https://doi.org/10.18046/j.estger. 2018.146.2662

Porras Barajas, N. (2017). Una mirada a la sostenibilidad en la gestión de proyectos. Revista Daena (International Journal of Good Conscience), 12(3), 328-344. Obtenido de http://www.spentamexico.org/v12n3/A20.12(3)328-344.pdf

Robertson, M. (2017). Sustainability Principles and Practice (Segunda ed.). Routledge

Rodríguez Castilla, M. M., Peñaranda Peñaranda, M. M., y Manzano Duran, O. (2020). La Responsabilidad Social Empresarial: Un reto de las MiPymes. Bogotá, Colombia: Eco Ediciones

Sejenovich, H. (2020). Metodologías, instrumentos y conceptos para un desarrollo sustentable y socialmente justo. Obtenido de https://www.vocesenelfenix.com/con tent $/$ metodolog\%C3\%ADasinstrumentos-y-conceptos-para-undesarrollo-sustentable-y-socialmentejusto

Silvius, G., y Tharp, J. (2013). Susteainability integration for Effective Project
Management. IGI Global. doi:10.4018 / 978-1-4666-4177-8

World Commission on Environment and Development. (1987). Our common future. Obtenido de https://oliebana.files.wordpress.com/ 2012/09/1987-brundtland.pdf 